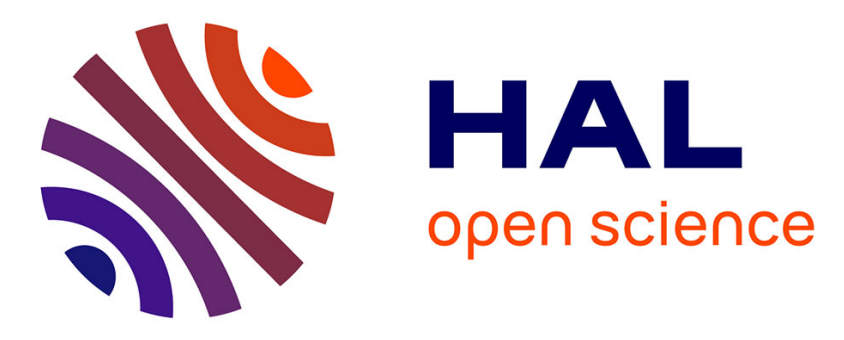

\title{
Understanding of others' knowledge in French and Japanese children: A comparative study with a disambiguation task on 16-38-month-olds
}

Hiroko Norimatsu, Raoul Blin, Kazuhide Hashiya, Christine Sorsana, Hiromi Kobayashi

\section{To cite this version:}

Hiroko Norimatsu, Raoul Blin, Kazuhide Hashiya, Christine Sorsana, Hiromi Kobayashi. Understanding of others' knowledge in French and Japanese children: A comparative study with a disambiguation task on 16-38-month-olds. Infant Behavior \& Development, 2014, pp.632-643. hal-01099820

\section{HAL Id: hal-01099820 https://hal.science/hal-01099820}

Submitted on 24 Jun 2018

HAL is a multi-disciplinary open access archive for the deposit and dissemination of scientific research documents, whether they are published or not. The documents may come from teaching and research institutions in France or abroad, or from public or private research centers.
L'archive ouverte pluridisciplinaire HAL, est destinée au dépôt et à la diffusion de documents scientifiques de niveau recherche, publiés ou non, émanant des établissements d'enseignement et de recherche français ou étrangers, des laboratoires publics ou privés. 


\title{
Understanding of others' knowledge in French and Japanese children: A comparative study with a disambiguation task on 16-38-month-olds
}

\author{
Norimatsu, H. ${ }^{1}$, Blin, R. ${ }^{2}$, Hashiya, K. ${ }^{3}$, Sorsana, C. ${ }^{4} \&$ Kobayashi, H. ${ }^{5}$ \\ 1. CLLE-LTC (Cognition, Langues, Langage, Ergonomie_Laboratoire Travail \& Cognition)—UMR 5263, University \\ of Toulouse, Toulouse, France \\ 2. Centre National de la Recherche Scientifique, Centre de Recherches Linguistiques sur l'Asie Orientale-UMR 8563, \\ Paris, France \\ 3, 5. Faculty of Human-Environmental Studies, Kyushu University, Fukuoka, Japan \\ 4. University of Toulouse and University of Lorraine, (InterPsy Laboratory, E.A. 4432, Nancy 2), France
}

\begin{abstract}
In order to explain the cultural differences reported in the results of false-belief tasks, we attempted to verify the 'task bias hypothesis' suggested by certain studies (e.g. Tardif, Wellman, \& Cheung, 2004; Rubio-Fernandez \& Geurts, 2013). At the same time, we aimed to observe the Theory of Mind (ToM) ability of infants and young children under the age of three in verbal communication. To this end, we propose a new protocol to test young children's ToM ability, with particular attention paid to the linguistic aspect of the task. This original disambiguation task using proper nouns (first names) was tested on a total of 32 children aged between 16-38 months, in France and Japan. The results revealed that after the age of 30 months children begin to correctly interpret nouns while simultaneously taking into account their partner's knowledge $(50 \%$ of the French and $29 \%$ of the Japanese children were successful), whereas this remains difficult for younger children (no child under 30 months was successful). The analysis of error types has shown that 'memory bias' was dominant in younger children in particular and 'association bias' was rarely observed across all ages. Given that the results of French and Japanese children did not differ significantly, we assume that this new task design could minimise the influence of cultural difference caused by the characteristics of different languages.
\end{abstract}

Key words: Theory of Mind, Language, Disambiguation task, French, Japanese, Young children

\section{1- Introduction}

\subsection{Cultural differences in the acquisition of Theory of Mind}

Over the past thirty years much research has been devoted to investigating when infants or young children acquire the ability to understand others' mental states, or 'Theory of Mind' (ToM). While Premack \& Woodruff's (1978) first definition of ToM involved the understanding of a wide range of mental states, such as the desires, goals, intentions, knowledge and beliefs of others, much developmental research on human children has focused essentially on false-belief understanding using the standard false-belief task (Wimmer \& Perner, 1983), or a variation thereof. If we examine the results of a large number of studies that use the false-belief task, we can see a discrepancy in the 
age at which different countries report a majority of children succeeding in this task (e.g. Wellman, Cross, \& Watson, 2001; Liu, Wellman, Tardif, \& Sabbagh, 2008). According to these results, some delay has been reported in Asian children (Chinese, Korean and Japanese) (Lewis, Koyasu, Oh, Ogawa, Short, \& Huang, 2009; Lewis, Huang, \& Rooksby, 2006; Tardif, Wellman, \& Cheung, 2004; Liu, Wellman, Tardif, \& Sabbagh, 2008), in particular Japanese children (Naito \& Koyama, 2006; Okumura, Moriguchi, Kanokogi, \& Itakura, 2009, etc.). Japanese studies have found that the majority of children succeed in this task at around 5.5 to 6 years old, compared to 4 years old as generally reported in European or North-American children. No clear explanation for this cultural discrepancy has yet to be provided. However, two hypotheses have been formulated by some researchers.

1) The first hypothesis concerns the influence of cultural environment on the development of ToM (e.g. Liu, Wellman, Tardif, \& Sabbagh, 2008; Lewis, Koyasu, Oh, Ogawa, Short, \& Huang, 2009; Kazama, Hirabayashi, Karasawa, Tardif, \& Olson, 2013). In this view it is assumed that children's experience in their daily sociocultural context could affect their performance in understanding other people's mental states.

2) The second hypothesis concerns the existence of a methodological or protocol bias, including linguistic bias, in the task (e.g. Rubio-Fernandez \& Geurts, 2013; Tardif, Wellman, \& Cheung, 2004). Rubio-Fernandez \& Geurts (2013), for example, have highlighted many distractors in the standard false-belief task which prevent young children under 4 years of age from remaining focused and cause them to fail in the task. In other words, a child's true ToM ability might be underestimated due to task bias. (Rubio-Fernandez \& Geurts (2013) proposed a new version of the false-belief task that allows children to visually track the protagonist and 3-year-olds were highly successful in the task). In addition, the differences induced by translations may be included in such methodological bias. Tardif et al. (2004) reported a significant difference in the results of Chinese children in the false-belief task according to the verbs used. This reveals an important problem of equivalence of the phrases used in the task.

Moreover, a combination of these two hypotheses exists: Naito \& Koyama (2006) reported that many Japanese children interviewed after the false-belief task answered that they had focused their attention on peripheral elements of the story rather than the main point in order to understand the false-belief of others. This directing of their attention to other elements may partly explain their less successful results in the task.

We focused on the second hypothesis of protocol bias since the first hypothesis concerning cultural environment poses a difficulty in identifying the candidate factors potentially affecting ToM development (see Liu et al., 2008).

The hypothesis of protocol bias encompasses two issues: 1) it could be a source of the discrepancy between false-belief task results in different countries; 2) it could be a barrier to examining the ToM ability of younger children under 3 years of age as the task requires a certain degree of linguistic ability. In an effort to find a solution to these problems we will suggest a new protocol to measure ToM ability in younger children under 3 years of age, with particular attention paid to the linguistic aspect of the task.

To our knowledge there are few studies that combine children's linguistic ability, their sociocognitive ability (ToM ability) and cultural comparison. We attempt to do this in the present study.

\subsection{Theory of Mind in younger children under the age of three}

The standard false-belief task requires a minimum level of language ability in children and becomes difficult to test on younger children under 3 years of age. In the false-belief task, even though the child's answer may be a simple verbal or gestural one, the question asked implies the 
comprehension of complex embodied structures like 'The child thinks that A will do B because A believes that the object is still in the first location where (s)he placed it'. Furthermore, the ability to interpret this kind of verbal statement structure requires a certain degree of language ability and may be a barrier to examining ToM understanding in younger children under three.

In order to explore the ToM ability of young children and infants, some studies in the last decade have used non-verbal tasks (Call \& Tomasello, 1999; Onishi \& Baillargeon, 2005; Southgate, Senju, $\&$ Csibra, 2007, etc.). As for the type of response used by the child or infant, previous studies have employed different methods: some studies used gaze behaviour to evaluate infants' ability (Onishi \& Baillargeon, 2005; Southgate, Senju, \& Csibra, 2007, etc.), while others used non-verbal behavioural responses (Call \& Tomasello, 1999; Buttelmann, Carpenter \& Tomasello, 2009) or a combination of both (O'Neill, 1996). The results in young infants using gaze behaviour have led to a new hypothesis on false-belief understanding, provoking heated debate; however, some researchers consider gestural or verbal responses by toddlers and young children to be more powerful or explicit evidence of their understanding of mental states (Clements \& Perner, 1994; Call \& Tomasello, 1999; Apperly \& Butterfill, 2009; Buttelmann, Carpenter \& Tomasello, 2009). It is for this reason that we have chosen to work on ToM ability in speech acts or 'verbal communication'.

Moreover, since verbal communication is a fundamental mode of human communication, it is our view that even for infants or young children, not integrating the verbal mode into the methodology would be prejudicial to improving our understanding of human social cognition. Our challenge was to propose a new protocol based on verbal communication which allows ToM ability in younger children to be observed. Recent research on referential communication has demonstrated the possibility of observing young children's ability to take into account others' knowledge in verbal communication (Campbell, Brooks \& Tomasello, 2000; Matthews, Lieven, Theakston, Tomasello, 2006; Salomo, Graaf, Lieven \& Tomasello, 2011) and revealed that 3- and 4 years-olds are capable of choosing different referring expressions (noun vs. pronouns) depending on their knowledge of others. Recently, Gundel and Johnson (2013) used their analysis of a corpus of natural discourse to observe children aged from 21 to 32 months and their ability to choose nouns, pronouns or demonstratives according to the knowledge of their partner. However, these studies examined young children's ability in verbal 'production' (of different referring expressions) and this could be a barrier to observing the ability of younger children under 2.5 years of age. Given that comprehension precedes production in language acquisition, we conclude that in order to study younger children we must opt for a protocol that uses an 'interpretation task' in speech acts. In this line, Gross, Moll \& Tomasello (2010) observed how infants of 21 months interpret sentences containing the articles 'the' and ' $a$ ' in a context where they have to take into account the cooperative logic of requests. Similarly, by using an interpretation task, Ganea \& Saylor (2007) succeeded in studying children as young as 15 months. This study revealed that 15 -month-olds' behaviour in interpretation was influenced by the fact that the experience of others had been taken into account. While this result is encouraging, we suspect some bias in the procedure. Indeed, the procedure contained three parts: familiarisation, absent reference and test. The first phase consisted in familiarising the infant with the test objects, rooms and the testing procedure. The experimenter talked about her desire to find one of the test objects. She then asked the infant to help her find it and indicated the next room as the location of the object. She invited the infant to go and find it by asking, 'Can you get the shoe?'. Depending on the infant's response (correct or not), the experimenter gave positive feedback or corrected the child. We cannot exclude the possibility that the infant was trained by this familiarisation phase to simply take the object that had been named several times when the experimenter pointed to the next room. Subsequently, during the absent reference phase the infant heard E1 mention an 'absent objet' 8 times and E1 involved the infant in finding it together. Following this phase, the test consisted in asking the infant to find the object in the next room using only a pronoun, 'I know where it is! It's here!' Then the experimenter coded the object chosen by the infant. If the expected one was chosen, the infant was considered to have 
understood the pronoun 'it'. However, the infant may simply have chosen the object that had been named many times, even if (s)he did not understand the function of the pronoun. To exclude this eventuality, we would need to see what happened without words, just by just saying 'I know', for example, using no pronouns and simply pointing to the next room. If the same result was obtained using this method, we could conclude that the pronoun 'it' had no effect. It is thus impossible to conclude (or exclude) that the child used ToM to interpret the pronoun 'it' in this experiment.

Even though this study contains some potential problems, it shows an interesting line of research and we expect that observing non-verbal responses in an 'interpretation task' will enable us to explore the ability of young children under 2.5 years.

\subsection{Relationship between language ability and ToM}

Studies on the relationship between language ability and ToM can be classified into three categories. 1) The first includes studies in which language ability is not taken into account and language merely serves as a 'tool' in the ToM task. Much research on ToM in children falls into this category. 2) The second category places special emphasis on the relationship between children's language ability and that of ToM, but uses separate measures for these two skills. Numerous studies fall into this category (see the meta-analysis by Milligan, Astington, \& Dack, 2007). In this approach, ToM ability is evaluated using the standard false-belief task and verbal language abilities are evaluated using various language developmental scales or tests. The relationship between the results of the different tests is then analysed at a later stage. 3) The third category of studies also focuses on the relationship between language ability and ToM; however, these two types of ability are examined simultaneously in one task. Our protocol falls into this category.

Among the studies in which both ToM and language abilities are handled together in the same task, two sets of approaches can be distinguished. The first of these approaches assumes that some words or structures of words reflect a specific mental state. Consequently, by observing the use of these words, it is possible to identify which mental state is concerned. Research adopting this approach comprises both monolingual studies (e.g. Lee, Olson, \& Torrance, 1999) and multilingual studies (e.g. Matsui, Rakoczy, Miura, \& Tomasello, 2009). For example, Matsui et al. (2009) observed children's ability to understand a speaker's level of certainty when this level is explicitly verbalised with a modal marker ('yo', 'kana' in Japanese).

The second approach differs from the previous one by focusing on the child's ability to use ToM in the processing of language. Following a commonly held point of view in linguistic pragmatics and semantics, it assumes that verbal language is highly underspecified (meaning that we say much less than we understand). In order to decode or interpret a verbal message (for example a sentence), we use a considerable amount of extralinguistic information known as 'contextual' information. We assume that contextual information includes beliefs about others, in other words, that speech acts depend on ToM ability. There are very few studies that explicitly explain how ToM is involved in language processing. Two of the studies mentioned above which use an interpretation task follow this approach. One such study is by Ganea \& Saylor (2007). They suppose that to correctly use the pronoun 'it' as they observed, it is necessary for the interpreter - the infant - to 'infer that the object the experimenter wanted was the one that [the experimenter] had previously talked about'.

Another study is that by Gross, Moll \& Tomasello (2010). They studied whether 12- and 18-monthold infants can understand the logic of cooperative requests. Their protocol consists in observing the interpretation of a request for help. They varied the articles 'the' and ' $a$ ' in the request sentence ('Give me the battery' or 'Now you can do it. Take $a$ battery') and the situation of the requester (hands free vs. hands occupied). The context was made ambiguous with two possible referred objects (e.g. two batteries) positioned at different distances from the requester (one reachable and another out of reach). The authors assume that the way the request is interpreted, in other words which object will be chosen, will depend on the infant's ability to understand the need for help and their ability to assess which object is most useful to the requester. 
In our study, we propose to observe how children will disambiguate a proper noun by taking into account (their belief concerning) the locutor's knowledge. Like the study by Ganea \& Saylor (2007), we will first explain how proper nouns are connected to ToM. Indeed, according to a theoretical model conceived by observing the processing of interpretations of proper nouns in adults discourse (Blin, 2009), the child will have to take into account logical reasoning on the knowledge of others. Furthermore, the child will have to identify new information for the interlocutor and himself, and also distinguish between the knowledge of two different partners. We will describe this theoretical model in detail below.

\subsection{Linguistic bias in the case of cultural comparison}

We will focus on the linguistic facts that may make it difficult to observe ToM ability in young children or which may be a source of cultural bias. To do so, we will present some examples in French, Japanese and English.

Although a large number of morpho-syntactical structures are common to many languages (socalled 'linguistic universals'), their frequency and familiarity are not necessarily the same. To avoid cultural bias it is necessary to choose structures with comparable familiarity in the compared cultures. A great number of studies in the field of referential communication focus on the use of pronouns, articles and demonstrative determiners (Gundel \& Johnson 2013; Matthews, Lieven, Theakston, \& Tomasello, 2006, etc.). Rubio-Fernandez and Geurts (2013), for example, used sentences containing many pronouns in their task. In Japanese, however, and particularly in the language of young children, pronouns are not frequently used. It is more usual to call people by their name. It is very natural even for young children to refer to themselves using their first name. Pronouns are thus better avoided when comparing Japanese with French or English.

The complexity of the sentences used in a task may make it difficult to observe the ability of younger children or infants. For example, verbs of belief often require the use of subordinate clauses (for example sentences like I think 'that he $V$ '), and such complex sentences are not used by very young children (according to the example given by Diessel (2004), 'I think' appears in production at around the end of the third year). It is also necessary to control the complexity of the sentences to avoid cultural bias. For example, with verbs of belief, the verb in the subordinate clause sometimes needs to be conjugated in the subjunctive in French. This conjugation is not fully mastered before elementary school age (e.g. Clark, 1985). It is clear that the difficulty in manipulating complex sentences is quite different in French and Japanese. Consequently, in order to observe young children's linguistic ability and avoid cultural bias in the context of a comparative study (in this case between French and Japanese), we assume that it is preferable to use sentences that are syntactically very simple.

In spite of these precautions, a significant difficulty relating to the expression of 'quantification' remains, particularly when comparing Japanese to European languages, for example. It is for this reason that we have chosen to focus on proper nouns in our task instead of common nouns. The following difficulties may be highlighted.

In Japanese, unlike in French or English for example, it is not necessary to systematically make quantification explicit. Many common nouns thus appear in sentences without any quantificational marker (such as 'a', 'the' or 'some' in English). To simplify, unlike French sentences where all common nouns have an explicit quantifier, it can be noted that Japanese quantification is ambiguous (underspecified or unspecified). When we translate the instructions in an experiment from French or English to Japanese, then, it is difficult to obtain exactly the same instructions in both languages. To illustrate this point we can take the example of the instruction 'Give me the battery' in the study by Gross et al. (2010). This sentence contains the quantifier 'the' of 'battery' which introduces the determination. In this context 'the' signals that the expected battery is 'known to both the locutor and the interpreter' and is necessarily one of the two batteries on the table. Furthermore, the singular form of 'battery' (instead of the plural form 'batteries') specifies the number. This means 
that one and only one battery is expected. Let us see what happens when translating this instruction into Japanese.

The most natural Japanese sentence (in this context) would be 'batteri choudai' (battery Object give). This sentence does not contain any quantification marker. Thus, in this context 'batteri' could be interpreted either as 'one of the batteries' or 'both of them'. In another context 'batteri' could also designate any existing battery in the world. Compared to the original sentence in English, the quantification in the Japanese sentence is clearly underspecified and its effects on the interpreter are considerably different. The complication here is that it is quite difficult to obtain a similar natural sentence, for reasons we shall now examine.

- by introducing the numeral 'ikko' (one) we are sure to obtain the expected number but the definite meaning is lost: 'batteri ikko choudai' means 'any one' of those batteries rather than a 'specific one'.

- if a determiner (kono, sono, ano) is added: 'Sono batteri (ikko) choudai', we introduce a notion of proximality between locutor, interlocutor and the object, which is not present in the English determiner and which specifies which battery must be taken, given the position of the locutor. Any other translation would affect the simplicity and spontaneity of the original sentence and produce an unnatural sentence that would disturb the listener.

As we can see, the quantification of common nouns makes it difficult to obtain sentences that are exactly equivalent in English/French and Japanese. And Japanese is not the only exception. Many other languages underspecify quantification. This is the case for example with Mandarin and Korean.

One further reason which guides us to avoid the use of quantification markers is that the use of determiners appears only after 2.5 years of age, in specific contexts (Bassano, 2000), and full adultlike use is not observed before the age of 7 (Clark, 1985).

Despite this, nouns (as a morpho-syntactical category) should not be avoided because they are used by children at a very early developmental stage, before the mastering of verbs (Gentner, 1978). Using nouns makes it possible to observe the ability of infants or very young children. One solution is to use nouns that do not require any quantificational marker. This is the case of proper nouns. In the following section we will present our new protocol using proper nouns and explain why such a verbal protocol is interesting for the study of ToM.

\section{2- A disambiguation task using proper nouns (a new protocol)}

In our new protocol we focused on how to observe Theory of Mind by analysing the child's ability to disambiguate the locutor's sentence. In this task the child must disambiguate a proper noun which could refer to two different characters.

We will first explain how proper nouns are concerned by Theory of Mind and why the use of proper nouns is interesting. Secondly, we will present the key features of the protocol and then situate it with regards to other existing ones.

\subsection{Interpreting proper nouns using Theory of mind}

It is assumed that in a collaborative discourse in context (Grice, 1979) the interpreter clarifies an ambiguous proper noun (without a quantificational marker) by taking into account his/her belief about the knowledge of the generator (Blin, 2009). We can briefly summarise the process of interpreting proper nouns as follows.

In our everyday life it is very common to know different persons with the same first name. However, in discourse and in context the first name will designate only one of these persons. In order to find to whom a first name refers, the interpreter will choose one and only one person among the persons 
s/he knows and among those s/he believes the locutor knows. We will call the persons having the same first name and known by a given interpreter his/her 'referring values of this first name'. In other words, for a name which appears in discourse the interpreter will choose one person who belongs to both his own set of referring values and the set of referring values he attributes to the locutor. It is thus necessary for the interpreter to possess a belief about the other's knowledge.

The focus of our protocol is to determine whether a child, when hearing a first name with multiple possible referring values, will or will not use his belief about the locutor's knowledge to disambiguate this first name in context.

The use of proper nouns, and especially that of first names, is interesting for the study of ToM ability. First of all, they are used frequently at all ages, making a cross-age experiment possible. Secondly, they allow us to observe ToM ability in young children because proper nouns are used and heard from a very early developmental stage and the syntax is very simple. Thirdly, they may potentially minimise cultural bias in the task because their syntactic properties are comparable in many languages. Additionally, and in particular, they (commonly) appear 'without any quantification makers' (vs. Protocol of Grosse, Moll, \& Tomasello (2010) using the articles 'the' or ' $a$ ' in the key sentences addressed to the child).

\subsection{States of mind during the disambiguation task}

In this section we will present the protocol and describe how it is able to evaluate the mental state of the interpreter-child at each stage. A detailed version of the task will be presented in the following section on method.

The protocol is divided into four stages. We used two puppets P1 and P2, both named Popi. Two adults E1 and E2 took part in the experiment.

During the first stage, a puppet P1 is introduced by the experimenter E1 while experimenter E2 is absent. Consequently, the child knows this puppet $\mathrm{P} 1$ and his name. In other words, the referring value of Popi for the child is the puppet P1. S/he also knows that E1 knows puppet P1 and his name.

During the second stage the second adult E2 enters and introduces the second puppet while E1 is absent. Thus, the child knows this puppet P2 and his name. In other words, the referring value of Popi for the child is P1 and P2. S/he also knows that E2 knows puppet P2 and his name.

In the third stage E2 puts all the puppets into a box and closes it while the child is looking. E1 then returns and $\mathrm{E} 2$ leaves.

In the fourth stage the child is in the presence of the two homonymous puppets and only one experimenter (E1). E1 asks for 'Popi' without giving any information that could guide the child or specify which one of $\mathrm{P} 1$ and $\mathrm{P} 2$ is expected. The point is to observe which puppet will be chosen by the child.

According to the model of interpretation of proper nouns (Blin 2009), if the child uses his/her belief about the other's knowledge, s/he should pick up the puppet P1 that has been introduced by the locutor/adult (E1) because s/he didn't have the information that the locutor knows the other puppet (P2).

\section{3- Method}

\subsection{Participants}

Sixteen French and sixteen Japanese children aged from 16 to 38 months (mean age: 27.4 months, 
all were boys) participated in the experiment. As our aim was to compare two cultural groups (French vs. Japanese) and age ranges (16-38 months), we preferred not to add a third variable, 'gender', to this sample. An additional two children were excluded from the analysis because of their fussy state due to shyness. Five additional children aged four to six years participated in the study in order for us to observe the reaction of older children to the protocol.

\subsection{Material}

Four animal puppets (an elephant, a rabbit, a monkey and a frog), two of which share the same first name, a mini-car, a piece of toy cake and a box. The choice of puppets was based on their familiarity as animals and the size of the puppets was chosen to allow infants or young children to reach and take hold of them easily.

The experiment was conducted in a quiet separate room at a daycare centre in France and in an experiment room (with childish decoration) at a university in Japan. All the experiments were videotaped facing the child.

\subsection{Detailed procedure}

\section{Familiarisation play time:}

To familiarise the child with the experimenters and the surroundings, the child and a parent were invited to the experiment room where two experimenters played with the child for about 15-20 minutes. During this familiarisation time an experimenter explained the research conditions to the parent once again and filled out the consent form.

\section{Phase 1:}

Each child was seated on a chair beside their parent (or on the parent's lap) in front of a table. Experimenter 1 was seated on the other side of the table.

Experimenter 1 (E1) tells a short, simple story to the child using two puppets. E1 begins by introducing two puppets named 'Popi' (rabbit puppet) and 'Tom' (elephant puppet) along with their characteristics. The story unfolds as follows: 'Today Popi is playing with a car' (E1 makes the noise 'Brooom, broom') 'and suddenly Popi falls over'. “'Ouch!” Popi can't stand up'. 'Just then, Tom arrives' and helps Popi to get up (using gestures). Popi says 'Thank you'. Tom replies 'You are welcome' and leaves the scene. E1 says, 'The story is finished and Popi says "Good-bye"'. Popi then leaves the scene. Immediately after this story, E1 swops the two puppets over (left-right) under the table and then places them on the table asking the child, 'In this story, which one was Popi?' If the correct answer is given E1 says, 'Yes, you're right. And who is the other one?' If the child's answer is incorrect E1 says, 'No, this is Popi. And that one? That is Tom'. In any case, E1 presents the two puppets again and confirms their correct names.

After this, E1 says, 'Oh, I have to go to the toilet. Could you wait a little? I'll be back soon, all right?' and leaves the room. At the same time, experimenter 2 (E2) enters the room with two other puppets saying, 'I'll tell you another story while E1 (E1's name) is not here'.

\section{Phase 2:}

Experimenter 2 (E2) begins telling another story with the two remaining puppets. E2 introduces two puppets named 'Popi' (monkey puppet) and 'Lea' (frog puppet) along with their characteristics. In this second story E2 says, 'Today Popi is jumping' (E2 makes Popi (the monkey) jump), 'Now Popi is hungry. Popi wants to eat some cake'. 'Just then, Lea arrives with a cake' and gives it to Popi. 
Popi says 'Mmmm, it's tasty. Thank you'. Lea says, 'You are welcome' and leaves the scene. E2 says, "The story is finished, Popi says "Good-bye"" and Popi leaves the scene. In the second story, the monkey puppet has the same name ('Popi') as the rabbit puppet from the first story. Immediately after this story E2 swops the two puppets over (left-right) under the table, places them on the table and asks the child, 'In this story, which one was Popi?' and if the correct answer is given E2 says, 'Yes, you're right. And the other one, what's their name?' If the child's answer is incorrect, E2 says, 'No, this is Popi. And that one? That is Lea'. In any case, E2 presents the two puppets again and confirms their correct names.

\section{Phase 3:}

Experimenter 2 (E2) puts all four puppets in a box saying, 'All the friends are going to sleep now,' and closes the box. The position of the four puppets in the box was decided in advance (see Figure 1-c) and the same for all children. At this point E1 returns and E2 leaves the room saying, 'As E1 is back I'll go now. Good-bye.'

\section{Phase 4:}

Once E1 has returned and is seated in front of the child she asks the first question: 'Oh, where is Popi?' and observes the child's reaction. Generally the child indicates (verbally or non-verbally) the closed box holding all the puppets. To this reaction E1 says, 'Oh, here? In this box?' then opens it towards the child and asks him the second question, 'Can you give me Popi?', whilst holding out a hand to receive it. This question is asked while the child is facing the 'four puppets' in the box, whereas E1 cannot see them because of the lid of the box. The reaction of the child is observed and coded.

We would like emphasise that when E1 asks the child this question, E1's gesture (holding out a hand) is not redundant because while it does have a meaning, it is highly underspecified compared to the verbal question. Indeed, although holding out a hand could be understood as meaning 'give me', it does not point to any particular object. It could then be interpreted as meaning 'give me something'. The choice of a specific puppet in the box cannot be determined by this gesture. Consequently, the child's choice depends on their interpretation of the noun.

Insert Figure 1-a, Figure 1-b \& Figure 1-c here

Color figures have to be reproduced in "color on the Web" and in "black-and-white in print".

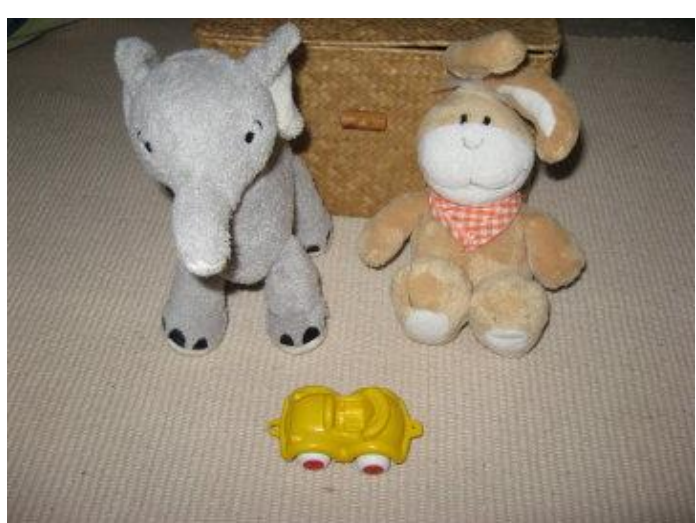

Figure 1-a. Tom \& Popi

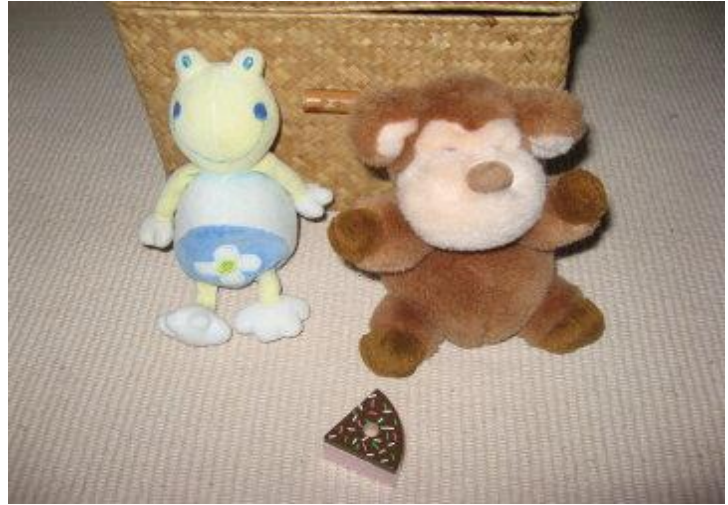

Figure 1-b. Lea \& Popi 


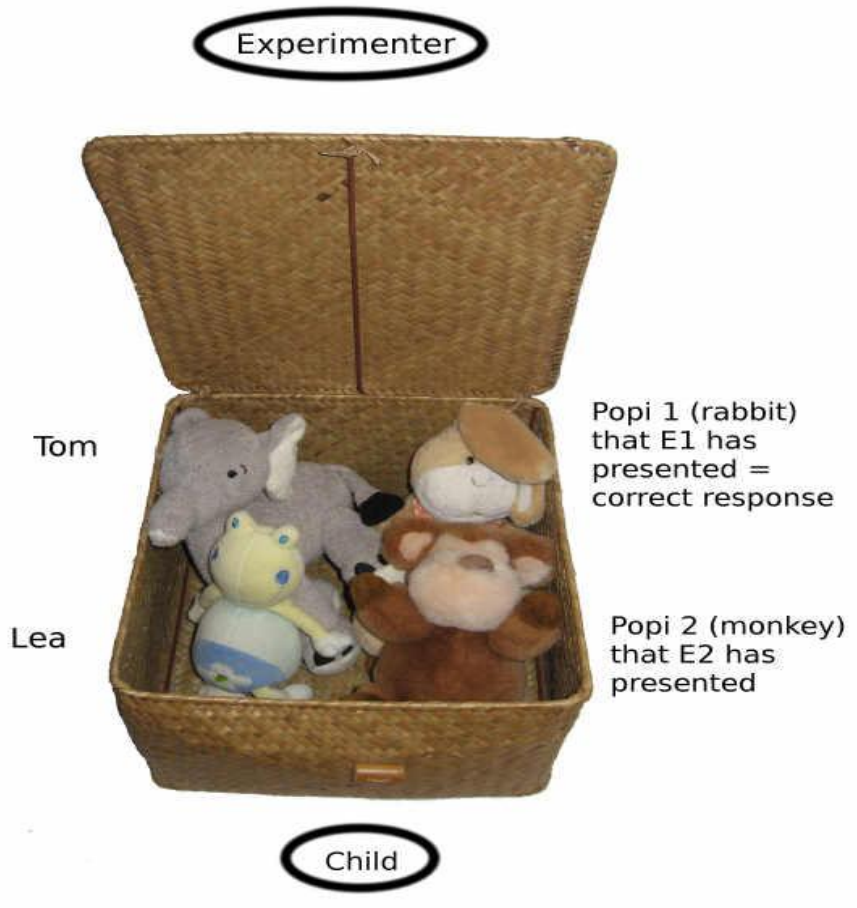

Figure 1-c. The position of the four puppets in the box.

\subsection{Coding}

The child's behavioural reaction to the three questions was analysed.

For the first question, 'Where is Popi?', we observed if the child indicated the location of the puppet or not.

For the second question, 'Can you give me Popi?', there were five possible reactions from the child to this request: 1) The child picks up the Popi that E1 knows (correct referring value of the first name \& taking into account of E1's knowledge); 2) the child picks up the two puppets that E1 used (incorrect referring value of the first name but taking into account E1's knowledge); 3) the child picks up another Popi that E1 does not know (correct referring value of the first name but not taking into account E1's knowledge); 4) the child picks up two puppets named Popi (P1 \& P2) (correct referring values of the first name but not disambiguating the multiples values); and 5) other choices of puppet(s). In the case of the child choosing a puppet other than the correct Popi, E1 replies, 'Hmmm, is this Popi?' (the third question) and observes the child's reaction. If the child picks up two puppets, including the correct Popi, E1 asks, 'Which one is Popi?'

The coding of the children's behavioural response was done by two independent persons. The rate of agreement was $97 \%, 97 \%$ and $88 \%$ respectively for the three questions, and Cohen's kappa was $\mathrm{k}=0.78,0.95$, and 0.83 . The cases of disagreement were discussed in order to specify the criteria for the final coding. 


\section{Results}

\subsection{Results of the children's first response}

All of the children except two French and two Japanese children indicated the closed box as the location of the requested puppet (to the question 'Where is Popi?').

\subsection{Results of the second question}

Once the location of the puppet had been indicated, the response to the request 'Give me Popi' was categorised into five possibilities. Apart from one younger French child, all the children picked up an object to indicate a response physically to the question. Two younger French children picked up objects other than the four puppets (invalid response). The child's first response to the request out of five possible answers was analysed. The results of children in both countries are shown in Figure 2. No child under the age of 30 months, in either country, gave the correct answer (correct referring value of the first name and taking into account of E1's knowledge). As for the children aged between 30-38 months, two out of seven Japanese and four out of eight French children gave the correct answer. In both countries we noted the appearance of a correct response after 30 months. However, the difference between the two countries on children over the age of 30 months (proportion of correct and incorrect responses) is not significant $\left(1, \chi^{2}\right.$ with Yates' correction $=$ $1.01, \mathrm{n} . \mathrm{s})$.

Insert Figure 2 here

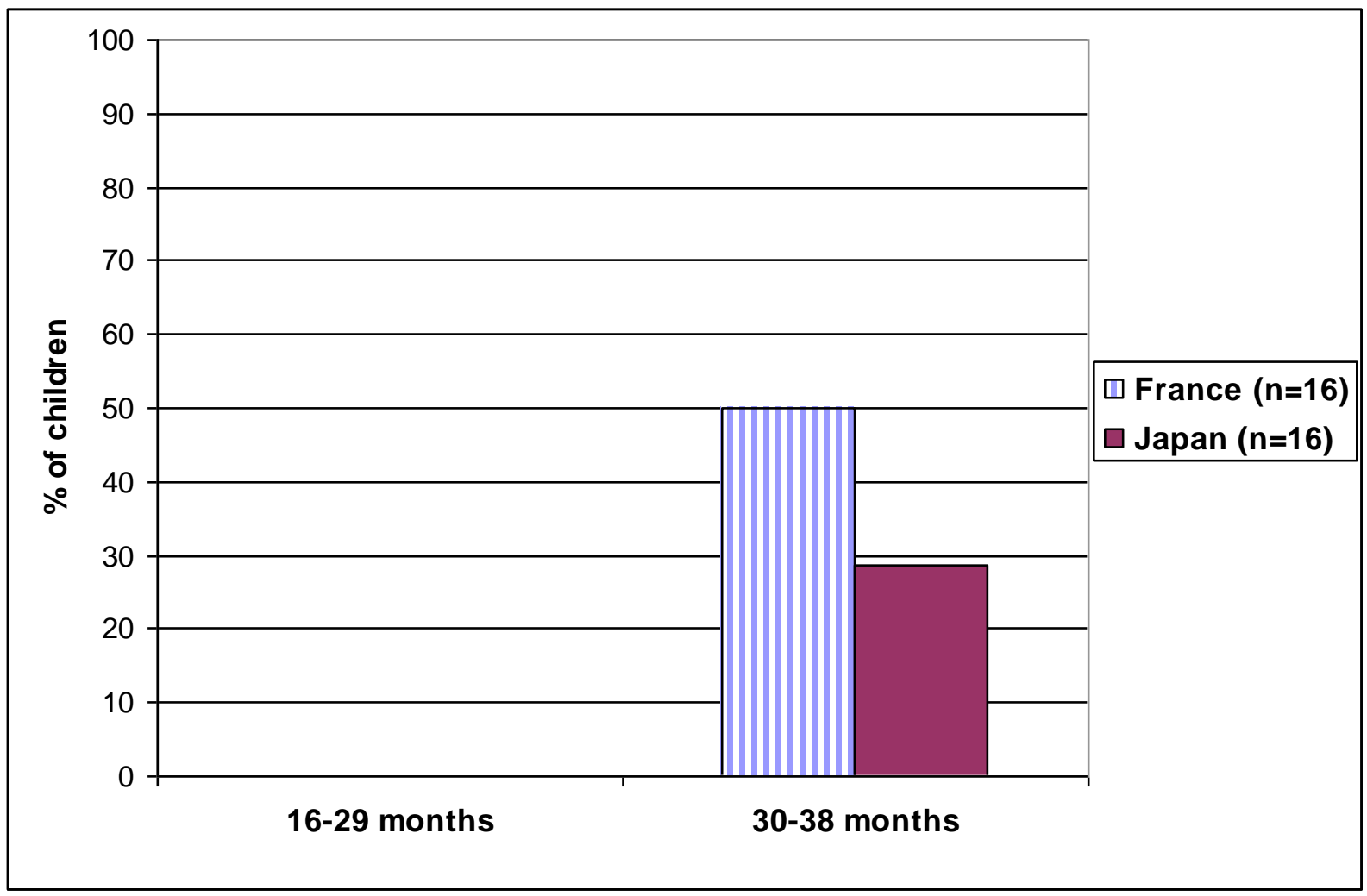

Figure 2: Percentage of correct responses according to age group and culture. 


\subsection{Results of error patterns}

In order to examine the error patterns of the responses, five patterns of answers were analysed. P1: The child picks up the Popi known to E1 (correct referring value of the first name and taking into account of E1's knowledge), P2: the child picks up the two puppets that E1 used (answer using E1's knowledge only), P3: the child picks up another Popi unknown to E1 (correct referring value of the first name but not taking into account of E1's knowledge), P4: the child picks up the two puppets called Popi (referring value is correct but child does not disambiguate the two referring values), P5: other choices of puppet(s) (neither correct referring value of the first name nor taking into account E1's knowledge), and 'Not valid': responses including 'no response' or 'touching an object other than the four puppets'. The results of the five patterns are shown in Table 1 and Figure 3. The dominant error type was P3: 'another Popi', which indicates a correct referring value for the first name 'Popi' but the perspective of the first experimenter is not taken into account. This could be considered 'latest memory bias' because 'another Popi' was memorised in the last story told by the second experimenter. This type of error constituted the majority of younger children's responses, though it decreased after the age of 30 months. At the same time, the percentage of correct responses increased (taking into account the locutor's knowledge + correct referring value of the first name). Association type error (P2: taking into account only the locutor's knowledge) was infrequent in all age groups. It is notable that the error type (P4) was absent (picking up two puppets called Popi), and that the error type (P5) was also not observed (no child picked up only the frog or only the elephant or combinations other than the previous answer patterns). All children except six picked up one puppet only, and this one puppet was either the correct Popi or the incorrect Popi. These results could indicate that young children understand from a very early stage of development that first names refer to only one value. Taking others' knowledge into account becomes possible later, enabling older children to answer correctly.

Insert Table 1 here

Table 1. Number of children in the five response patterns in France and Japan.

\begin{tabular}{|l|c|c|c|c|c|c|}
\hline \multicolumn{1}{|c|}{$\begin{array}{l}\text { Type of } \\
\text { answer }\end{array}$} & $\begin{array}{l}\text { Correct } \\
\text { response }\end{array}$ & \multicolumn{5}{|c|}{ Error types } \\
\hline $\begin{array}{l}\text { P1: } \\
\text { First name \& } \\
\text { locutor's } \\
\text { knowledge }\end{array}$ & $\begin{array}{l}\text { P2: } \\
\text { Locutor's } \\
\text { knowledge } \\
\text { only }\end{array}$ & $\begin{array}{l}\text { P3: } \\
\text { First name } \\
\text { only }\end{array}$ & $\begin{array}{l}\text { P4: } \\
\text { First name but } \\
\text { does not } \\
\text { disambiguate }\end{array}$ & $\begin{array}{l}\text { P5: } \\
\text { Other }\end{array}$ & Not valid \\
\hline $\begin{array}{l}\text { FR; 16-29 } \\
\text { months (n=8) }\end{array}$ & 0 & 1 & 4 & 0 & 0 & 3 \\
\hline $\begin{array}{l}\text { JP; } \mathbf{1 6 - 2 9} \\
\text { months (n=9) }\end{array}$ & 0 & 1 & 8 & 0 & 0 & 0 \\
\hline $\begin{array}{l}\text { FR; } 30-38 \\
\text { months (n=8) }\end{array}$ & 4 & 3 & 1 & 0 & 0 & 0 \\
\hline $\begin{array}{l}\text { JP; } 30-38 \\
\text { months (n=7) }\end{array}$ & 2 & 1 & 4 & 0 & 0 & 0 \\
\hline
\end{tabular}

Note: P1) The child picks up the Popi known to E1 (correct referring value of the first name and taking into account of E1's knowledge), P2) the child picks up the two puppets that E1 used (answer using E1's knowledge only), P3) the child picks up another Popi unknown to E1 (correct referring value of the first name but not taking into account of E1's knowledge), P4) the child picks up the two puppets called Popi (referring value is correct but child does not disambiguate the two referring values), and P5) other choices of puppet(s) (neither correct referring value of the first name nor taking into account E1's knowledge); Not valid = response including 'no response' or 'touching an object other than the four puppets'. 
Insert Figure 3 here

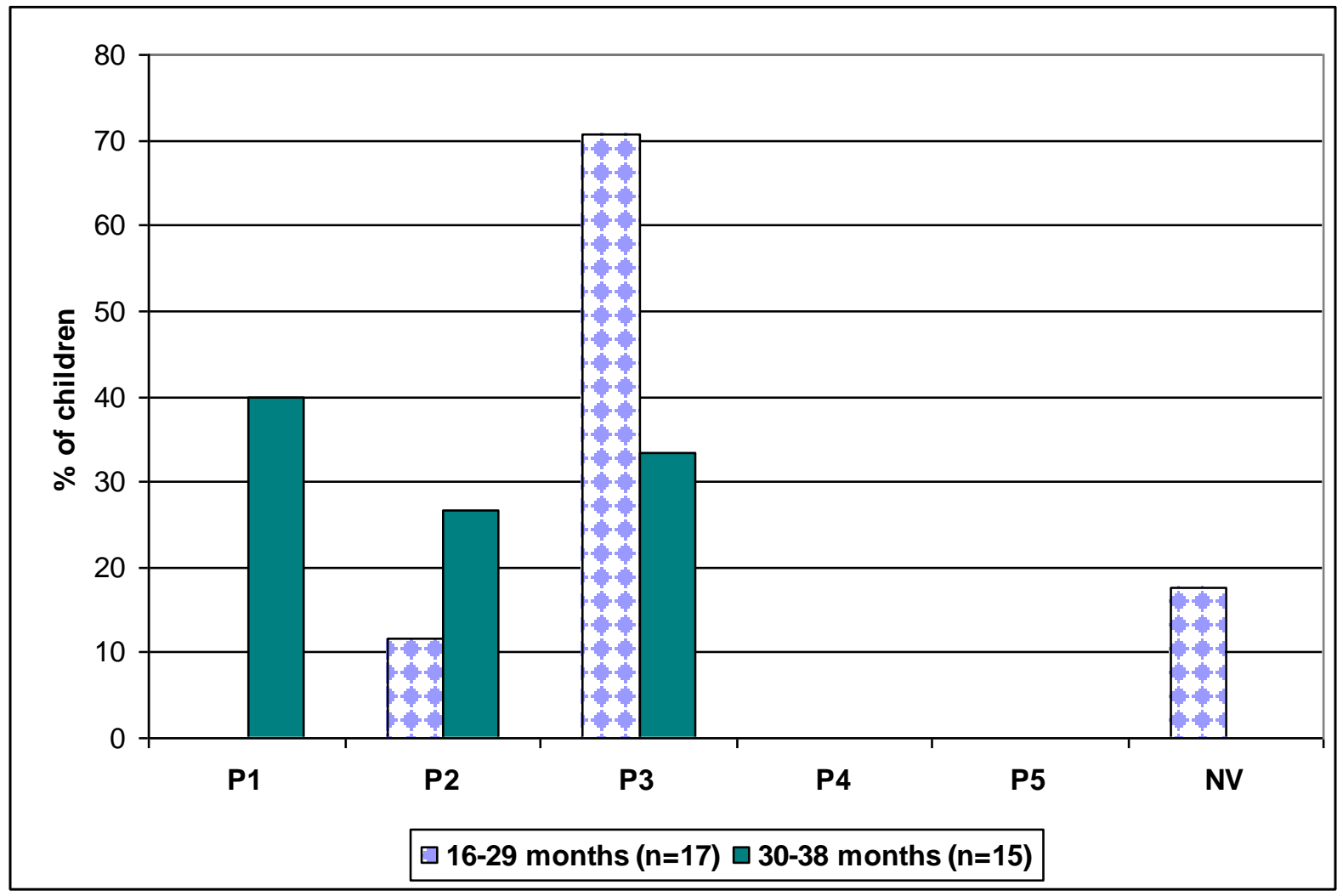

Figure 3: Response pattern percentages according to age group (French and Japanese children combined).

Note: P1) The child picks up the Popi known to E1 (correct referring value of the first name and taking into account of E1's knowledge), P2) the child picks up the two puppets that E1 used (answer using E1's knowledge only), P3) the child picks up another Popi unknown to E1 (correct referring value of the first name but not taking into account of E1's knowledge), P4) the child picks up the two puppets called Popi (referring value is correct but child does not disambiguate the two referring values), and P5) other choices of puppet(s) (neither correct referring value of the first name nor taking into account E1's knowledge); NV) Response not valid including 'no response' or 'touching an object other than the four puppets'. 


\subsection{Analysis of the reaction to the third question}

The third question posed in the case that the child's first response (to the second question) was incorrect allowed the experimenter to verify the state of the child's memory. This question varied according to the first response of the child. We analysed the reaction to this question.

In the case that the child picked up the Popi that E2 had used, E1 asked a third question, 'Is this Popi?', with an attitude of surprise. This pattern (P3) was the most frequent error (latest memory bias) and 17 children fell into this category. Eight of them picked up the other Popi (correct response) when E1 asked the third question. This indicates that the first response was based on the latest memory but the name of the other Popi puppet had not been forgotten. In response to this third question two children picked up the two puppets that E1 had used (by association, including the correct Popi) and seven children picked up other puppets or all the puppets including the correct Popi. The last case in which the child picked up other puppets may indicate that he had forgotten which one was the first Popi. This reaction was frequent in younger children (6 of the 7 cases were in children under 30 months).

In the case that the child picked up the two puppets used by E1 (including the correct Popi, P2: association bias) as their first response, E1 asked 'Which one is Popi?' as the third question. Four of the six children in this category indicated Popi (correct response) to this question, while one of them explained spontaneously that two puppets had the same name and pointed at the two target puppets (Popi 1 \& Popi 2).

\section{Discussion}

The present study aimed to explore the ability of young children to understand others' knowledge. We proposed a new verbal communication-based protocol which could minimise the influence of different languages in the case of cultural comparison. We tested our new disambiguation task on samples of French and Japanese boys aged 16 to 38 months. In further studies, it could be tested with girls.

By using this protocol, our study could be placed at the intersection of two fields: 'theory of mind' and 'referential communication'. Although we observed ToM ability in young children, our research differs from other ToM studies in the particular attention paid to linguistic data in the task. On the other hand, the present study falls into the realm of referential communication because the task involves interpreting the referring value (meaning) of a noun in context, or more precisely, according to the knowledge of the interlocutor. While interest in ToM in the field of referential communication seems to have increased over the last decade, few studies clearly explain the specificities of linguistic data and their link to ToM, as we have already pointed out. The present study should be seen as one of these rare studies. One of the particularities of our study lies in its use of an interpretation task, because the majority of referential communication studies focus on children's ability in production. Our interpretation task allows us to observe the ability of younger children under the age of three in verbal communication and in ToM simultaneously.

With this protocol the mean duration of the experiment was around five minutes. This short and simple task allowed us to observe the ability of younger children to disambiguate the locutor's sentence using ToM. At the same time, the results of the older children (4-6 years) on whom we tested this protocol were all successful. The discussion with these older children after the experiment informed us that the task (in their eyes, two short stories) seemed pleasant to them and not boring (too easy) or strange. Some of them asked to continue with other stories. This suggests that the task could be adapted for a wide range of ages and could be used on one- to six-year-olds or older. 
Our results with 16-38-month-olds reveal that children aged over 30 months begin to show signs of interpreting proper nouns according to their belief about the knowledge possessed by their partnerinterlocutor. The appearance of a correct response after the age of 30 months was observed in both the French and Japanese groups.

Before arriving at the stage of giving a correct response, an analysis of the error types has shown that the dominant pattern was 'Correct referring value of the first name but partner's knowledge not taken into account'. In this case, the child picked up a puppet named with the requested noun, but not the right one in relation to the locutor's knowledge. This pattern may be influenced by the latest memory information, since the puppet picked up by the child had been presented in the second and last story. The pattern consisting of 'the two puppets associated with the partner' was rarely observed. It is notable that no child picked up two puppets called Popi, and that all children showed responses that included only one of the two Popis (right Popi or wrong Popi). These findings show that young children understand the characteristics of proper nouns, as referring to only one individual/object, even in the absence of any linguistic quantifier indicating that there is only one. Other possible errors such as 'only picking up a puppet named differently to the one requested' was not observed at all.

In the case of the child choosing the wrong Popi puppet, when the first experimenter expressed her surprise the children picked up the other Popi puppet (i.e., gave the correct response) more frequently than the two other possibilities (puppets with other first names). Firstly, this behaviour shows that the child is able to detect an abnormality in his/her interpretation according to the locutor's reaction. This phenomenon of auto-correction suggests pragmatic competences at a very early age. What is more, despite the large amount of information to keep in mind during this task two characters in story 1 , plus two more characters in story 2, with a same first name for two of them and new first names for the others -, these results indicate that the puppets' names were relatively well memorised. The children's reaction to the third question suggests that latest memory bias could be more relevant than association bias (i.e., the child chooses the two puppets associated with E1).

These results will guide us to explore the memory bias hypothesis in further studies using this protocol. In the past, the question of children's working memory capacity in relation to their performance on standard false-belief tasks have been examined (Davis \& Pratt, 1995; Gordon \& Olson, 1998; Perner \& Lang, 1999). However, with the present protocol we need to address the question of memory capacity at a much earlier developmental stage (between 1 to 3 years of age) compared to that of the standard false-belief task (generally 3 to 6 years of age). As our results have shown (Figure 3), memory bias-type error in the present protocol was much more frequent in 16-29month-olds (71\%) compared to 30-38-month-olds (33\%). Consequently, the issue of memory capacity must be examined in children under the age of 30 months in particular. Furthermore, the question of source memory, studied as a related ability to ToM (Naito, 2003; Lind \& Bowler, 2009), may be a further interesting element to test in relation to our protocol.

\section{Question of association}

We adopted two strategies to minimise the problem of the simple association of puppetexperimenter in the responses of children. First of all, we used two puppets for each experimenter. This meant that by simple association between puppets and experimenter there were always two possible puppets, one of which was called Popi. However, when the child was asked to respond to the question 'Can you give me Popi?' all four puppets were presented to the child. When the child picked up the correct Popi among the four puppets, s/he could do so by association with E1; however, s/he also had to use a further criterion, the referring value of the first name 'Popi'. Our results ultimately showed that few children (only 6 children out of 32) showed the error pattern of using association only by picking up the two puppets that E1 had used. 
The second strategy was to ask a third question, 'Which one is Popi?', when the child picked up the two puppets associated to E1 in response to the request 'Can you give me Popi?' This third question allowed us to observe what happened in this case. Among the six children who picked up the two puppets that E1 had used, four could indicate immediately which one was Popi out of the two puppets. Therefore, it could be considered that such an answer satisfies the first experimenter's request, because in proposing the two puppets used by E1 the child includes the right Popi puppet; and it is possible that this could be a case of over-interpretation of E1 wanting to find her puppets again (because they had disappeared from E1's sight). Given that the study by Grosse, Moll \& Tomasello (2010) has shown that 21-month-olds are able to understand the cooperative logic of requests in a very simple sentence from their interlocutor, our results could be interpreted as indicating that at least four of the six children who picked up the two puppets used by E1 had understood not only which puppet was requested, but also E1's desire to find her puppets again. One of these four children explained spontaneously that two puppets had the same name, and that the other two puppets were different (in terms of their names). Another child picked up the two puppets used by E1 and explained spontaneously, 'The other two puppets belong to another woman'. According to the behavioural criteria of our coding their responses were coded as 'error response'; however, we could not deny the possibility that we had underestimated their comprehension.

To summarise, the results of the present study using a proper noun disambiguation task reveal that after the age of 30 months children begin to interpret proper nouns while simultaneously taking into account their partner's knowledge, whereas this remains difficult for younger children. These results are quite similar to those of the study by O'Neill (1996). In both studies children around 30 months or older showed their understanding of their partner's knowledge (lack or novelty of) through an active behavioural response: in our study, children's choice of a puppet among four possibilities sometimes accompanied with a verbal response; and in O'Neill's study, verbal, gestural (pointing) and gaze behaviour. As for cultural differences, in the observed age range, the results of French and Japanese children did not significantly differ. We assume that the new task design may minimise the influence of cultural difference stemming from different language characteristics. In our introduction we mentioned that two hypotheses exist to explain the important cultural discrepancy reported previously in children's ToM ability: 1) the first hypothesis concerning the influence of cultural environment on the development of ToM, and 2) the second hypothesis of a methodological or protocol bias, including linguistic bias, in the task. The results of our research lead us to favour the second hypothesis of protocol bias. However, we cannot exclude the possibility of a difference that could appear after the age range observed in the present study. This must be verified through further research and tested in different countries-languages.

\section{Acknowledgements}

We are grateful to the children and their families for taking part in this study. We would like to address our thanks to the daycare centre and Centre Communale d'Action Sociale in Toulouse, and Baby Lab at Kyushu University for their help with this research. Our thanks also go to Michiru Ueno, Saki Yokoishi, Xianwei Meng, Kana Tajiri, Yuka Akiyoshi, Reiki Kishimoto for their assistance with data collection in Japan. We are also grateful to the anonymous reviewers for their helpful comments on the previous manuscript.

\section{Author Contributions}

Conception of the project: HN \& RB. Theoretical reviews: HN RB KH. Design of the experiment: RB HN ChS. Performing the experiments: HN ChS HK RB. Data analysis: HN RB. Writing the paper: HN RB. 


\section{References}

Apperly, I. A., \& Butterfill, S. A. (2009). Do humans have two systems to track beliefs and belieflike states? Psychological Review, 116(4), 953-970. doi:10.1037/a0016923

Blin, R. (2009). Introduction à la linguistique formelle. Paris: Hermès science publications: Lavoisier.

Buttelmann, D., Carpenter M., \& Tomasello M. (2009). Eighteen-month-old infants show false belief understanding in an active helping paradigm. Gognition, 112, 337-342.

Bassano, D. (2000). Early development of nouns and verbs in French: Exploring the interface between the lexicon and grammar. Journal of Child Language, 27(3), 521-559.

Call, J., \& Tomasello, M. (1999). A nonverbal false belief task: the performance of children and great apes. Child development, 70(2), 381-395.

Campbell, A.L., Brooks P., \& Tomasello M. (2000). Factors affecting young children's use of pronouns as referring expressions. Journal of Speech, Language and Hearing Research, 43(6), 1337-49.

Clark, E. V. (1985). Acquisition of Romance, with special reference to French. In Slobin, D.I. (Ed.), The crosslinguistic study of language acquisition, vol. 1. The data. Hillsdale, NJ: Lawrence Erlbaum Associates. pp. 687-782.

Clements, W. A., \& Perner, J. (1994). Implicit Understanding of Belief. Cognitive Development, 9 , 377-395.

Davis, H. L., \& Pratt, C. (1995). The development of children's theory of mind: The working memory explanation. Australian Journal of Psychology, 47(1), 25-31.

Diessel H. (2004) The Acquisition of Complex Sentences. Cambridge University Press.

Ganea, P.A., \& Saylor M.S. (2007). Infants' Use of Shared Linguistic Information to Clarify Ambiguous Requests. Child Development, 78(2), 493-502.

Gentner, D. (1978). On relational meaning: The acquisition of verb meaning. Child Development, 49, 988-998.

Gordon, A.C. \& Olson, D.R. (1998). The relation between acquisition of a theory of mind and the capacity to hold in mind. Journal of Experimental Child Psychology, 68(1), 70-83.

Grosse, G., Moll, H., \& Tomasello, M. (2010). 21-Month-olds understand the cooperative logic of requests. Journal of Pragmatics, 42(12), 3377-3383. doi:10.1016/j.pragma.2010.05.005

Grice, H. P. (1979). Logique et conversation, Communication, 30, 57-72.

Gundel K.J., \& Johnson K. (2013). Children's use of referring expressions in spontaneous discourse: Implications for theory of mind development. Journal of Pragmatics, 56, 43-57.

Kazama, M., Hirabayashi, H., Karasawa, M., Tardif, T., \& Olson, S. (2013). Ambiguous parenting and four-year-olds' understanding of others: A comparison between mothers in Japan and the U.S. The Japanese Journal of Developmental Psychology, 24 (2), 126-138. (in Japanese).

Lee, K., Olson, D. R., \& Torrance, N. (1999). Chinese children's understanding of false beliefs: the role of language. Journal of Child Language, 26(1), 1-21.

Lewis, C., Koyasu, M., Oh, S., Ogawa, A., Short, B., \& Huang, Z. (2009). Culture, executive function, and social understanding. In C. Lewis \& J. I. M. Carpendale (Eds.), Social interaction and the development of executive function. New Directions in Child and Adolescent Development, 123, 69-85.

Lewis, C., Huang, Z., \& Rooksby, M. (2006). Chinese preschooler's false belief understanding: is social knowledge underpinned by parental styles, social interactions or executive functions? Psychologia -An International Journal of Psychology in the Orient, 49(4), 252-266. doi:10.2117/psysoc.2006.252

Lind, S.E. \& Bowler, D.M. (2009). Recognition memory, self-other source memory, and theory-ofmind in children with autism spectrum disorder. Journal of Autism and Developmental Disorders, 39(9), 1231-1239.

Liu, D., Wellman, H. M., Tardif, T., \& Sabbagh, M. A. (2008). Theory of mind development in Chinese children: a meta-analysis of false-belief understanding across cultures and languages. 
Developmental Psychology, 44 (2), 523-531.

Matthews D., Lieven E., Theakston A., \& Tomasello M. (2006). The effect of perceptual availability and prior discourse on young children's use of referring expressions. Applied Psycholinguistics, 27, 403-422.

Matsui, T., Rakoczy, H., Miura, Y., \& Tomasello, M. (2009). Understanding of speaker certainty and false-belief reasoning: a comparison of Japanese and German preschoolers. Developmental Science, 12(4), 602-613. doi:10.1111/j.1467-7687.2008.00812.x

Milligan, K., Astington, J. W., \& Dack, L. A. (2007) Language and theory of mind : Meta-analysis of the relation between language ability and False-belief understanding. Child Development, 78 (2), 622-646.

Naito, M. (2003). The relationship between theory of mind and episodic memory: evidence for the development of autonoetic consciousness. Journal of Experimental Child Psychology, 85(4), 312-336.

Naito, M., \& Koyama, K. (2006). The development of false-belief understanding in Japanese children: Delay and difference? International journal of behavioral development, 30(4), 290-304.

Okumura, Y., Moriguchi, Y., Kanokogi, Y., \& Itakura, S. (2009). Kokoro no riron kenkyuu ni taisuru hi-gengo kadai no yuukousei - Nihon to chuugoku no kodomo no hikaku kara -. (Efficacity of non-verbal task in the research on Theory of Mind - from a comparison of Japanese and Chinese children-). Paper presented in $9^{\text {th }}$ Japanese Conference on Baby Studies, May 16-17, 2009, Hikone, Japan.

O’Neill, D. K. (1996). Two-year-old children's sensitivity to a parent's knowledge state when making requests. Child Development, 67, 659-677.

Onishi, K. H., \& Baillargeon, R. (2005). Do 15-month-old infants understand false beliefs? Science, 308(5719), 255-258. doi:10.1126/science.1107621

Perner, J. \& Lang, B. (1999). Development of theory of mind and executive control. Trends in Cognitive Sciences, 3(9), 337-344.

Premack, D., \& Woodruff, G. (1978). Does the chimpanzee have a theory of mind? Behavioral and Brain Sciences, 1(4), 515-526. doi:10.1017/S0140525X00076512

Rubio-Fernandez, P., \& Geurts, B. (2013). How to pass the false-belief task before your fourth birthday. Psychological Science, 24(1), 27-33. doi:10.1177/0956797612447819

Salomo D., Graf E., Lieven E., \& Tomasello M. (2011). The role of perceptual availability and discourse context in young children's question answering. Journal of Child Language, 38, 918931.

Southgate, V., Senju, A., \& Csibra, G. (2007). Action anticipation through attribution of false belief by 2-year-olds. Psychological science, 18(7), 587-592. doi:10.1111/j.1467-9280.2007.01944.x

Tardif, T., Wellman, H. M., \& Cheung, K. M. (2004). False belief understanding in Cantonesespeaking children. Journal of Child Language, 31, 779-800.

Wellman, H. M., Cross, D., \& Watson, J. (2001). Meta-analysis of theory-of-mind development: The truth about false belief. Child Development, 72(3), 655-684.

Wimmer, H., \& Perner, J. (1983). Beliefs about beliefs: Representation and constraining function of wrong beliefs in young children's understanding of deception. Cognition, 13, 103-128. 